\title{
Hot Accretion onto Black Holes with Outflow
}

\author{
Myeong-Gu Park ${ }^{1, \star}$ and Du-Hwan $\mathrm{Han}^{1}$ \\ ${ }^{1}$ Kyungpook National University, Daegu 41566, KOREA
}

\begin{abstract}
Classic Bondi accretion flow can be generalized to rotating viscous accretion flow. Study of hot accretion flow onto black holes show that its physical charateristics change from Bondi-like for small gas angular momentum to disk-like for Keperian gas angular momentum. Especially, the mass accretion rate divided by the Bondi accretion rate is proportional to the viscosity parameter alpha and inversely proportional to the gas angular momentum divided by the Keplerian angular momentum at the Bondi radius for gas angular momentum comparable to the Keplerian value. The possible presence of outflow will increase the mass inflow rate at the Bondi radius but decrease the mass accretion rate across the black hole horizon by many orders of magnitude. This implies that the growth history of supermassive black holes and their coevolution with host galaxies will be dramatically changed when the accreted gas has angular momentum or develops an outflow.
\end{abstract}

\section{Introduction}

The amount of energy produced by the accretion is determined by the mass accretion rate $\dot{M}$ times the energy production efficiency $\varepsilon$. Black holes, however, do not have a hard surface, and the energy production efficiency is not given a priori unlike in accretion onto neutron stars or whited dwarfs. The efficiency is determined only by the detailed physical state and processes during the accretion, which depend critically on the mass accretion rate. The important question, yet often ignored, is what is the mass accretion rate onto black holes for general accretion flow such as rotating one and how does it depend on the physical parameters?

In this talk, the luminosity and the mass accretion rate are, respectively, normalized by the Eddington luminosity (for pure hydrogen accretion) $L_{E} \equiv 4 \pi G M m_{p} c / \sigma_{T h}=1.23 \times 10^{38}\left(M / M_{\odot}\right.$ ) ergs $/ \mathrm{s}$ and the Eddington mass accretion rate $\dot{M}_{E} \equiv L_{E} / c^{2}=2.2 \times 10^{-9}\left(M / M_{\odot}\right) M_{\odot} /$ yr where $G$ is the Gravitational constant, $c$ speed of light, $M$ mass of the accreting black hole, $m_{p}$ proton mass, and $\sigma_{T h}$ Thomson cross section. The dimensionless luminosity $l \equiv L / L_{E}$ and the mass accretion rate $\dot{m} \equiv \dot{M} / \dot{M}_{E}$ along with the radius in units of the Schwarzschild radius $r_{S c h} \equiv 2 G M / c^{2}$ make the problem scale-free with respect to the black hole mass, in most cases.

\section{Bondi Flow}

Bondi in his seminal work [1] studied spherical accretion of polytropic gas onto a point mass. He showed that four classes of solutions exist: supersonic, subsonic, transonic, and non-physical. Subsonic and transonic ones are the correct physical solutions for the real accretion: accretion flow may

^e-mail: mgp@knu.ac.kr 
stay subsonic all the way from outer-boundary to the stellar surface or starts subsonic, passes a sonic (critical) point, and becomes supersonic. Since black holes have no hard surface to build up enough pressure to slow down the infalling gas, transonic solutions are the relevant ones for accretion onto black holes. Bondi also realized that for given gas density and temperature at infinity, transonic accretion is possible only for a specific value of the mass accretion rate which is the maximum accretion rate. This mass accretion rate is called 'Bondi accretion rate',

$$
\dot{M}_{B}=\Lambda \gamma^{-3 / 2} 4 \pi r_{B}^{2} \rho_{\infty} c_{s, \infty}=4 \pi \Lambda \frac{(G M)^{2} \rho_{\infty}}{\gamma^{3 / 2} c_{s, \infty}^{3}},
$$

where $\rho_{\infty}$ is the mass density at infinity, $c_{s, \infty}$ the sound speed of gas at infinity, $r_{B} \equiv G M / c_{s, \infty}^{2}$ the Bondi radius, and $\Lambda$ is an order-of-unity function of the adiabatic index $\gamma$ [1]. This accretion rate has been widely used in almost any kind of accretion problems, perhaps too widely used.

\section{Rotating accretion flow}

Bondi's work dealt with accretion of gas with no angular momentum at all. But real cosmic accretion inevitably involves rotation. Park [2] used slim disk approximation (vertical structure of the disk is averaged) to study the behavior of the hot rotating accretion flow. Rotating accretion flow should have some kind of viscosity which transports the angular momentum outward, thereby enabling the inward accretion of gas; Park [2] adopted widely used alpha-viscosity prescription but in a simplified form. The equations contain a critical point (sonic point) as shown in Bondi flow [1] and slim disk [3], and the regularity condition at the critical point made the mass accretion rate an eigenvalue for given gas density, temperature, and angular momentum at the outer boundary. He found that the flow behavior changes from that of Bondi flow to that of disk flow as the gas angular momentum increases. Hot accretion flow constitutes a continuous family of solutions from non-rotating Bondi flow to almost Keplerian advection-dominated accretion flow (ADAF; [5-7]). The mass accretion rate also changed continuously from the Bondi accretion rate for small enough specific gas angular momentum at the outer boundary $J_{\text {out }} \lesssim 0.1 J_{B}$ ( $J_{B}$ is the Keplerian angular momentum at the Bondi radius), to an order-of-magnitude smaller rate for near Keplerian gas angular momentum (Fig. 1) with functional dependence

$$
\frac{\dot{M}}{\dot{M}_{B}}=\frac{\dot{m}}{\dot{m}_{B}}=0.09\left(\frac{\alpha}{0.01}\right)\left(\frac{J_{\text {out }}}{J_{B}}\right)^{-1} .
$$

Any gas that is fed to the central supermassive black hole in the real universe is likely to have non-negligible angular momentum, and the mass accretion rate can be much smaller than the classic Bondi accretion rate. This result has very important implications for the growth of black hole and black hole-galaxy coevolution: black holes accreting gas with near Keplerian angular momentum will grow with a factor of 10 smaller accretion rate than the Bondi accretion rate and, accordingly, the feedback from black hole to the host galaxy will be weaker.

Narayan \& Fabian [4] subsequently studied similar slowly rotating accretion flow onto a supermassive black hole to describe the cooling flow in a massive elliptical galaxy. They used alphaprescription viscosity proportional to the angular shear and slim disk approximation. They confirmed the result of [2] that the flow changes from Bondi-like to disk-like and the mass accretion rate decreases as $J_{\text {out }} / J_{B}$ increases. However, their $\dot{M} / \dot{M}_{B}$ versus $J_{\text {out }} / J_{B}$ has a much shallower slope than that of [2] and, therefore, the mass accretion rate decreases only to a factor of a few less than $\dot{M}_{B}$ even for a near-Keplerian flow. They suspected that the difference with [2] may be due to the different value of $r_{B}$ and suggested that a more thorough exploration of solutions in $\alpha-\left(J_{\text {out }} / J_{B}\right)-\left(r_{B} / r_{S c h}\right)$ is needed [4]. 

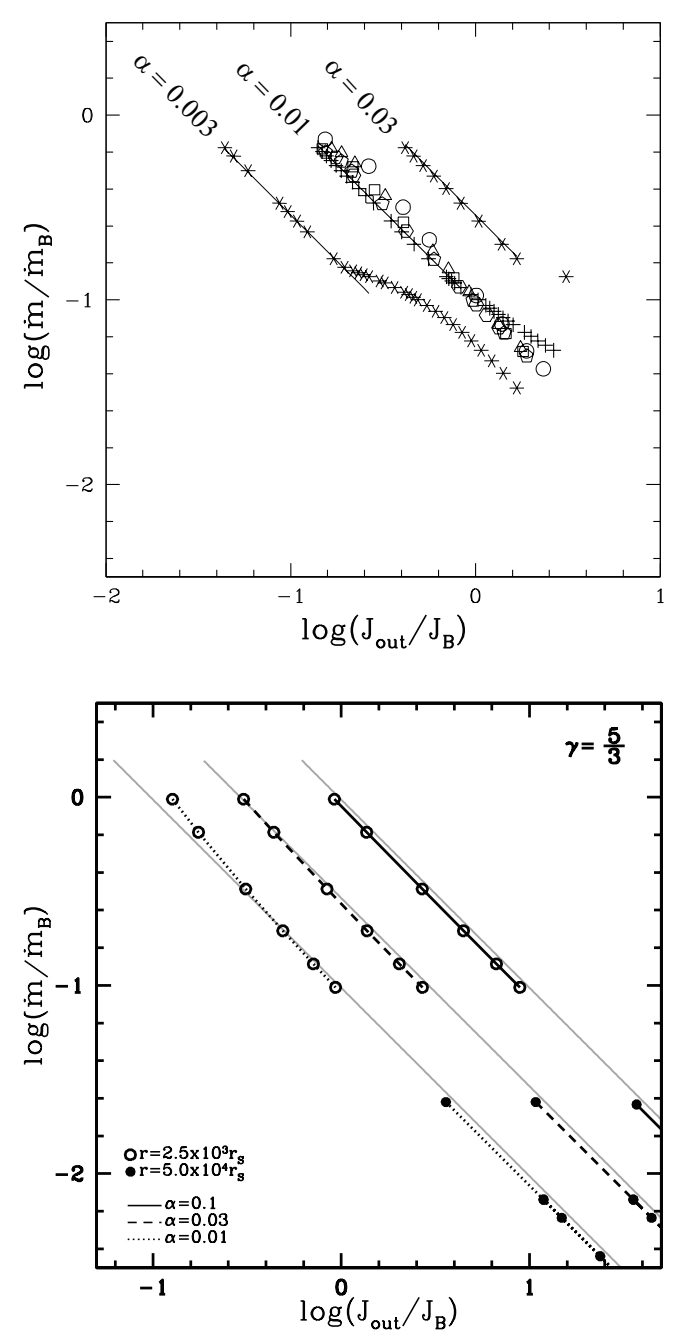

Figure 1. Mass accretion rate (in units of Bondi accretion rate) as a function of gas angular momentum (in units of Keplerian angular momentum) at the Bondi radius [2].
Figure 2. Mass accretion rate versus gas angular momentum for different values of the Bondi radius $r_{B}$ and the viscosity parameter $\alpha$.

\section{Generalized Bondi flow}

Following [2] and [4], we used slim disk approximation with alpha-description viscosity on the generalized Bondi flow, i.e., rotating polytropic gas, accreting onto a black hole. The adoption of polytropic gas equation of state will facilitate comparison with the original Bondi flow. We specifically focused on the dependence of flow characteristics on $r_{B}, \alpha$, and $J_{\text {out }} / J_{B}$ as suggested by [4].

Figure 2 shows the dependence of the mass accretion rate (in units of $M_{B}$ ) on the gas angular momentum at the Bondi radius (in units of $\left.J_{B}\right)$ for two different Bondi radii $\left(r_{B}=2.5 \times 10^{3} r_{S c h}\right.$, and $r_{B}=5.0 \times 10^{3} r_{S c h}$ and for three different values of viscosity parameter $(\alpha=0.1,0.03$, and 0.01$)$. All solutions show $\dot{M} / \dot{M}_{B} \propto\left(J_{\text {out }} / J_{B}\right)^{-1}$ (grey lines in Fig. 2 represent Eq. 2) seen in [2]. Different values of the Bondi radius, i.e., gas temperature, or viscosity parameter do not change the $\dot{M} / \dot{M}_{B^{-}}\left(J_{\text {out }} / J_{B}\right)^{-1}$ relation.

We also checked the dependence on the adiabatic index $\gamma$ of accreting gas. Figure 3 show the $\dot{M} / \dot{M}_{B^{-}}\left(J_{\text {out }} / J_{B}\right)^{-1}$ relation for different $\gamma$. The mass accretion rate ratio $\dot{M} / \dot{M}_{B}$ hardly changes for 


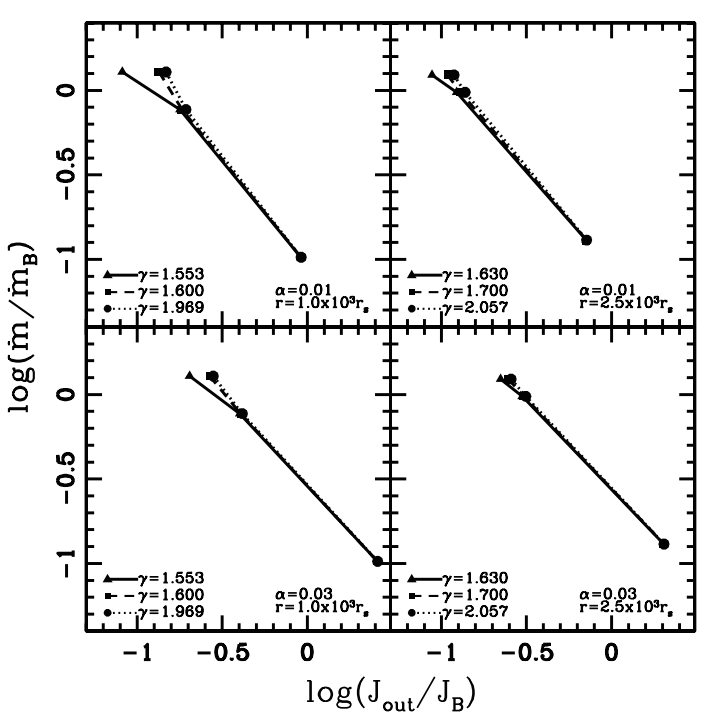

Figure 3. Mass accretion rate versus gas angular momentum for different values of the adiabatic index $\gamma$.

different values of $\gamma$, showing that $\dot{M}_{B}$ carries all the dependence on the adiabatic index even for generalized rotating Bondi flow.

So we can conclude that $\dot{M} / \dot{M}_{B^{-}}\left(J_{\text {out }} / J_{B}\right)^{-1}$ relation discovered in [2] is valid in an extended parameter space of $r_{B}, \alpha$, and $\gamma$.

\section{ADAF with Outflow}

As soon as the ADAF solutions appear, it was immediately realized that the ADAF has a positive Bernoulli constant [5]. This means that hot ADAF flows are very likely to develop an outflow or a wind. Blanford \& Begelman [8] proposed simple self-similar advection-dominated inflow-outflow solutions (ADIOS). In this models, hydrodynamic or magnetic wind, or equally outflow, carries off enough of the mass, angular momentum and energy to bind the gas to the black hole and to allow accretion to proceed [8]. That is, outflow helps maintain the accretion. Numerical simulations also show that outflow is common rather than exception, and the mass accretion rate through the inner boundary can be almost $1 / 100$ of the mass inflow rate from large radius [9].

We extended our calculation by implementing self-similar ADIOS of [8] to generalized Bondi flow to answer the question what is the mass accretion rate of the accreting flow in the presence of outflow. The equations contain similar critical structure seen in [2] and solved similarly. Figure 4 shows the resulting mass accretion rate $\dot{M} / \dot{M}_{B}$ of ADIOS versus ADAF as a function of gas angular momentum $\left(J_{\text {out }} / J_{B}\right)^{-1}$. The mass accretion rate of ADIOS are filled circles while those of ADAF open circles. ADIOS has a higher mass accretion rate than ADAF for the same gas conditions, including the gas angular momentum. This is because outflow removes angular momentum and the existence of outflow is equivalent to decreased gas angular momentum, and therefore, yields a higher mass accretion rate.

So we can conclude that outflows increase the mass accretion rate. However, the mass accretion rate here means the mass inflow rate at the outer boundary. In the presence of outflow, the mass accretion rate is not conserved and becomes a function of radius. In self-similar solutions of ADIOS, it is a power-law of radius [8], and the mass accretion rate at the inner boundary can be many factors of magnitude smaller than the mass inflow rate the outer boundary. Although the mass inflow rate increases by a factor of a few in the presence of outflow for given physical conditions of gas that 


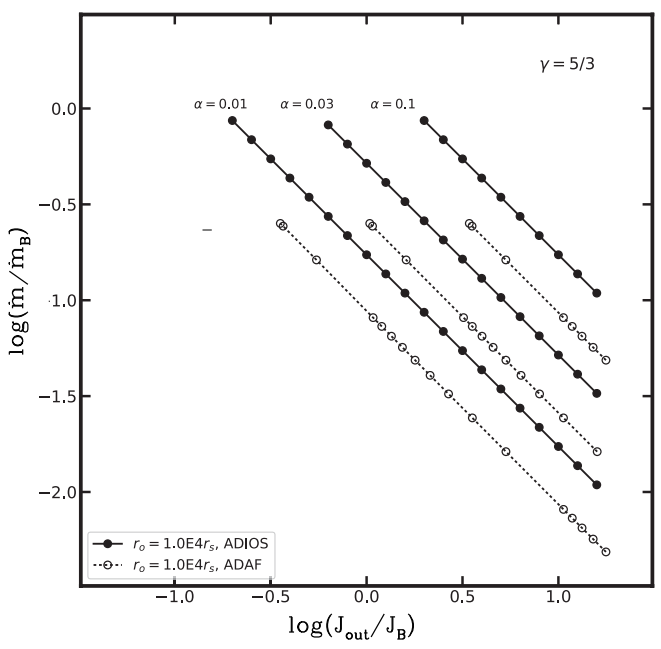

Figure 4. Mass accretion rate of ADIOS versus $\mathrm{ADAF}$ as a function of gas angular momentum.

surrounds the black hole, the final mass accretion rate that crosses the black hole horizon is much smaller by many orders of magnitude.

\section{Summary}

We have studied the structure and physical characteristics of polytropic rotating accretion flow onto black holes. We have confirmed that the mass accretion rate divided by the Bondi accretion rate is proportional to the viscosity parameter alpha and inversely proportional to the gas angular momentum divided by the Keplerian angular momentum at the Bondi radius, for a large parameter space of viscosity parameter alpha, gas temperature, and the adiabatic index. If a self-similar outflow is developed, the mass inflow rate at the outer boundary increases because the outflow carries away angular momentum but the mass accretion rate across the black hole horizon decreases by many orders of magnitude. The decrease in the mass accretion rate due to the gas angular momentum or outflow will have profound implications on the growth of supermassive black holes and their coevolution with host galaxies.

We thank the organizing committee of ICGAC-XIII/IK15 for hospitality. This research was supported by Kyungpook National University Research Fund, 2016.

\section{References}

[1] H. Bondi, MNRAS 112, 195 (1952)

[2] M.-G. Park, ApJ 706, 637 (2009)

[3] M. A. Abramowicz, B. Czerny, J. P. Lasota, and E. Szuszkiewicz, ApJ, 332646 (1988)

[4] R. Narayan and A. C. Fabian, MNRAS, 415372 (2011)

[5] R. Narayan and I. Yi, ApJL, 428 L13 (1994)

[6] R. Narayan and I. Yi, ApJ, 444231 (1995)

[7] R. Narayan and I. Yi, ApJ, 452710 (1995)

[8] R. D. Blandford and M. C. Begelman, MNRAS, 303 L1 (1999)

[9] J. Li, J. Ostriker, and R. Sunyaev, ApJ, 767105 (2013) 